\title{
Eribulin monotherapy in a patient with heavily pretreated metastatic breast cancer: Case study and review of the literature
}

\author{
Fariborz Gorouhi, Stefan Glück \\ Division of Hematology/Oncology, Department of Medicine, Sylvester Comprehensive Cancer Center, Miller School of \\ Medicine, University of Miami, Miami, Florida, USA \\ Correspondence: Stefan Glück. Address: Division of Hematology/Oncology, Department of Medicine, Sylvester \\ Comprehensive Cancer Center, Miller School of Medicine, University of Miami, 1475 NW 12th Avenue, Miami, FL 33136, \\ USA. E-mail: sgluck@med.miami.edu
}

Received: December 6, 2011

Accepted: December 4, 2012

Online Published: December 10, 2012

DOI : $10.5430 /$ jst.v3n1p21

URL: http://dx.doi.org/10.5430/jst.v3n1p21

\begin{abstract}
Treatment of heavily pretreated patients with metastatic breast cancer is challenging due to the combination of progressive disease and limited treatment options. As breast cancer in this setting is no longer curable, treatment goals include improvement/maintenance of quality of life, reduction of tumor-related symptoms, and prolonged survival. In the current review we discuss treatment options in late-line metastatic breast cancer, including newer chemotherapeutic agents such as eribulin, a recently approved non-taxane microtubule dynamics inhibitor whose mechanism of action is distinct from that of other tubulin-targeting agents. We also report on a 42-year-old woman with invasive ductal carcinoma (T4 N1 M1), positive for estrogen and progesterone receptors and negative for HER2, who had been heavily pretreated with radiation (to the bone metastases), hormonal therapy, and multiple systemic cytotoxic therapies-including a nanoparticle albumin-bound paclitaxel/bevacizumab/gemcitabine combination, and pegylated liposomal doxorubicin—none of which lasted for more than 4 months (due to progressive disease or toxicity). Upon receiving eribulin (in a clinical trial setting), she remained in stable disease for 21 months and generally tolerated eribulin well (except for thrombocytopenia, which resulted in mild, non-life-threatening gastrointestinal bleeding and was resolved with a dose reduction). This case is consistent with findings from phases 2 and 3 of eribulin in the setting of heavily pretreated metastatic breast cancer. It is discussed in the context of the current treatment goals and treatment modalities in the late-line metastatic breast cancer setting.
\end{abstract}

\section{Key words}

Metastatic breast cancer, Eribulin, Refractory, Anti-tubulin, Late-line setting

\section{I ntroduction}

Eribulin (E7389), a synthetic analog of halichondrin B (a natural polyether macrolide isolated from several marine sponges including Halichondria okadai), was developed to be structurally stable while retaining the anti-cancer bioactivity of the natural product ${ }^{[1]}$. Eribulin is a non-taxane microtubule dynamics inhibitor with a mechanism of action distinct from that of other tubulin-targeting agents. Eribulin binds exclusively to the growing plus ends of the microtubule, thereby 
suppressing dynamic instability ${ }^{[2]}$ through an inhibition of microtubule growth (with little to no effect on shortening), and causing sequestration of tubulin into nonproductive aggregates ${ }^{[3]}$.

Eribulin was approved in November 2010 by the US Food and Drug Administration (FDA) for the treatment of patients with metastatic breast cancer who had previously received at least 2 chemotherapeutic regimens for the treatment of metastatic disease. (Prior therapy should have included an anthracycline and a taxane in either the adjuvant or metastatic setting.) ${ }^{[4]}$

Herein we report the case of a patient with heavily pretreated metastatic breast cancer who received eribulin for approximately 21 months and discuss it in the context of current treatment goals and treatment modalities in the late-line metastatic breast cancer setting.

\section{Case study}

A 42-year-old woman presented with breast cancer (T4, N1, M1) in June 2008 (Figure 1). Two years earlier (2006), a breast mass was found and was treated as an abscess (because the initial biopsy result was negative) with incision and drainage (I\&D), as well as antibiotics, at another institution. Although this treatment was associated with some improvement, deterioration followed and the mass was treated again (in 2007) as an abscess with I\&D plus antibiotics. The wound did not heal; shoulder pain developed, followed by a bone fracture. Subsequently, a breast biopsy showed low-grade invasive ductal carcinoma, positive for both estrogen and progesterone receptors, and negative for HER2.

Figure 1. Timeline of events from initial discovery of breast mass to date. ABG, nanoparticle albumin-bound paclitaxel/bevacizumab/ gemcitabine regimen; RT, radiation therapy; TAM, tamoxifen.

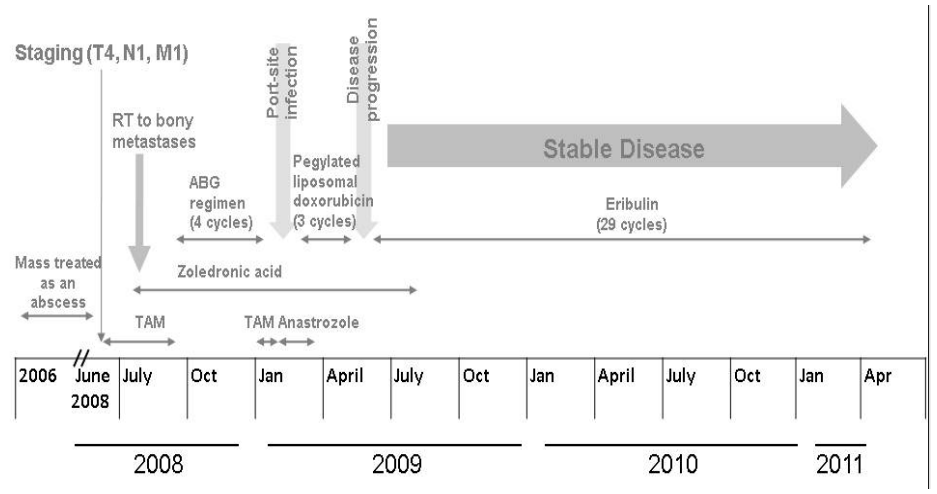

After presentation in our clinic and staging, the patient was treated with tamoxifen ( $20 \mathrm{mg}$ PO daily, from June 2008 through September 2008) and had a good response within 4 weeks (shrinkage of the tumor and closure of the skin lesion). In July 2008, she was started on zoledronic acid (4 mg intravenously [IV], every 4 weeks for 13 months) and received 3000-cGY radiation to the bone metastases (right humerus, pelvis, and right hip). In September 2008, tamoxifen was discontinued due to disease progression and the patient was started on 4 cycles of the ABG protocol (nanoparticle albumin-bound paclitaxel, $150 \mathrm{mg} / \mathrm{m}^{2} \mathrm{IV}$; bevacizumab $10 \mathrm{mg} / \mathrm{kg}$ body weight [BW] IV; gemcitabine, $1500 \mathrm{mg} / \mathrm{m}^{2} \mathrm{IV}$; on Days 1 and 15 of a 28-day cycle ${ }^{[5]}$. In December 2008, after 4 ABG cycles, an infection developed at the port site. Chemotherapy was stopped, and the infection was treated. In January 2009, tamoxifen therapy was begun (20 mg PO daily), but due to side effects (severe menopausal symptoms), treatment was switched to anastrozole (1 mg PO daily) 2 weeks later. The patient received anastrozole for less than 2 months due to worsening of bone metastasis pain.

In March 2009, the patient experienced progression of disease, with symptomatic bone metastases (despite stable findings on imaging studies), and was started on pegylated liposomal doxorubicin ( 3 cycles, $30 \mathrm{mg} / \mathrm{m}^{2} \mathrm{IV}$, on Days 1 and 15 of a 28-day cycle). By then, she had fully recovered from her port-site infection. Disease progression was detected again in 
May 2009; the patient experienced symptomatic bone metastases, and a bone scan showed progression of metastases and new lesions in the thoracic and lumbar spine and in the left acetabulum, whereas other lesions were unchanged (right humerus, right scapula, ribs) or decreased (calvarium) compared with previous imaging studies. Zoledronic acid was discontinued after 13 months of treatment because of bone pain. Due to disease progression, the pegylated liposomal doxorubicin was discontinued, and the patient was offered treatment in a clinical trial.

After the appropriate consenting process and fulfillment of eligibility criteria, the patient was randomized to receive Eribulin, which was initiated in June $2009\left(1.4 \mathrm{mg} / \mathrm{m}^{2}\right.$ on Days 1 and 8 of a 21-day cycle for the first 11 cycles) as part of the registered clinical trial NCT00246090. Due to thrombocytopenia, which resulted in minor rectal bleeding with a hemoglobin $(\mathrm{Hb})$ decrease of $2 \mathrm{~g} / \mathrm{dL}$ and hemodynamically stable, the dose was reduced according to protocol for all subsequent cycles to $1.1 \mathrm{mg} / \mathrm{m}^{2}$ ). The patient received a total of 29 cycles of eribulin as of June 10, 2012. Results of imaging studies obtained from July 2009 until June 2010 (bone scans and computed tomography [CT] scans of the chest, abdomen, and pelvis) demonstrated stable disease (SD) with no detectable progression.

Within 2 months of initiating eribulin therapy, the patient's pain subsided and has been minimal since October 2009 (with palliative treatment with long-acting opioids [fentanyl transdermal system] and short-acting pain medication taken as needed [including oxycodone, lidocaine transdermal patch, and ibuprofen]). The patient has been able to resume her normal daily activities, including college attendance.

Overall, the eribulin regimen was well tolerated by the patient, with complete blood count (CBC) and kidney function being normal since diagnosis. As per hospital protocol, the patient was premedicated with lorazepam $1 \mathrm{mg}$ PO, ondansetron $16 \mathrm{mg}$ IV, dexamethasone $10 \mathrm{mg}$ IV, and famotidine $20 \mathrm{mg}$ IV, with no specific premedication required for treatment with eribulin. While being treated with eribulin, the patient experienced minimal hypercalcemia (despite treatment with zoledronic acid) and mild hyperphosphatemia, which were not deemed to be associated with eribulin treatment. Serum levels were normal for intact parathyroid hormone (iPTH) and 25-hydroxy-vitamin D and were high-normal for 1,25-dihydroxy-vitamin D. (Vitamin D insufficiency was noted in November 2008 [prior to initiation of eribulin therapy in June 2009] and was treated with 50,000 U of vitamin D PO weekly for 8 weeks.) The patient developed no symptomatic side effects to eribulin treatment, with no reported incidence of alopecia or neuropathy.

\section{Discussion}

We report on the treatment of a patient with eribulin mesylate, an FDA-approved microtubule dynamics inhibitor. The time course of her treatment is illustrated in Figure 1 and demonstrates that she had been heavily pretreated (including hormonal therapy and a combination regimen of taxane/bevacizumab/gemcitabine as well as an anthracycline) prior to the initiation of eribulin therapy. Until eribulin was used, none of her systemic treatments had lasted longer than 4 months (due to progressive disease [PD] or toxicity). Following eribulin therapy, the patient's disease remained stable for 21 months.

Treatment of heavily pretreated patients with metastatic breast cancer remains a challenge. Furthermore, the late-line setting has become even more challenging over the years, as earlier and more aggressive use of chemotherapeutics in early lines limits the treatment options available in later lines, particularly as ideally, heavily pretreated patients require treatments that will not contribute to cumulative toxicity and will not exhibit cross-resistance with therapies previously used ${ }^{[6]}$. As metastatic breast cancer is no longer curable, primary goals of therapy in this setting and particularly in later lines of therapy include improving/maintaining quality of life, reducing tumor-related symptoms, and prolonging survival,

At present, no standard of care has been established for the late-line setting in metastatic breast cancer. The National Comprehensive Cancer Network (NCCN) guidelines provide an array of options for metastatic disease without recommending any particular regimen as superior in the late-line setting ${ }^{[7]}$. Notably, the NCCN guidelines do indicate that patients who had no response to 3 sequential chemotherapeutic regimens or for those with Eastern Cooperative Oncology 
Group (ECOG) performance status $\geqslant 3$, palliative care without further chemotherapeutics may be considered.7 The selection of chemotherapeutics in the late-line setting should be individualized and depend on several factors, such as patient and tumor characteristics (including comorbidities, drug resistance, chronic side effects), prior treatments, cumulative doses, availability, and the preference of the patient and physician ${ }^{[8]}$.

In recent years, more options for cytotoxic chemotherapy regimens have become available in the late-line metastatic breast cancer setting. For example, gemcitabine, a nucleoside metabolic inhibitor indicated in combination with paclitaxel for first-line treatment in metastatic breast cancer after failure of prior anthracycline-containing adjuvant chemotherapy (unless anthracyclines were clinically contraindicated) ${ }^{[9]}$, has also been evaluated (mostly in combination with other agents such as platinum-based chemotherapy or paclitaxel) in the metastatic breast cancer setting ${ }^{[10,11,12]}$. In addition, vinorelbine, a vinca alkaloid that interferes with microtubule assembly and is indicated in non-small cell lung cancer (NSCLC) ${ }^{[13]}$, has also been evaluated in the late-line metastatic breast cancer setting (as a monotherapy and in combination with capecitabine) ${ }^{[14,15]}$. Notably, the number of chemotherapeutics that are specifically indicated in late-line metastatic breast cancer has dramatically increased in recent years. This group of therapies now includes capecitabine (FDA-approved in 1998), nab-paclitaxel (FDA-approved in 2005), ixabepilone (FDA-approved in 2007), and the recently approved eribulin (FDA-approved in November 2010). Despite these advances, most of these agents (except for eribulin) did not demonstrate a survival benefit beyond second line in a randomized controlled trial.

Capecitabine is an orally administered antimetabolite indicated in combination with docetaxel for the treatment of patients with metastatic breast cancer after failure of prior anthracycline-containing chemotherapy and as a monotherapy for the treatment of patients with metastatic breast cancer resistant to both paclitaxel and an anthracycline-containing regimen or resistant to paclitaxel and for whom further anthracycline therapy is not indicated ${ }^{[16]}$. Capecitabine monotherapy was not compared to standard of care in comparative trials. In an open-label, single-arm trial, in patients previously treated with anthracyclines/taxanes, capecitabine monotherapy was associated with a response rate of $26 \%$, a median time to progression (TTP) of 3.3 months, and a median overall survival (OS) of 8.4 months in patients $(n=43)$ who were resistant to both paclitaxel and an anthracycline ${ }^{[16]}$. In a phase 3 study comparing capecitabine plus docetaxel to docetaxel monotherapy in 511 anthracycline-pretreated metastatic breast cancer patients (of whom approximately a third received study therapy as first-line treatment in metastatic disease and the remainder received study therapy as second- or third-line treatment for metastatic disease), median TTP and OS were significantly better in the docetaxel/capecitabine arm than in the docetaxel arm (6.1 vs. 4.2 months and 14.5 vs. 11.4 months, respectively; $P<0.05$ for both) ${ }^{[17]}$. The most frequent grade $3 / 4$ adverse events (AEs) in the capecitabine/docetaxel and docetaxel arms included neutropenic fever (16\% vs. $21 \%$, respectively), neutropenia ( $16 \%$ vs. $15 \%$, respectively), stomatitis ( $17 \%$ vs. $5 \%$, respectively), diarrhea ( $14 \%$ vs. $5 \%$, respectively), nausea ( $6 \%$ vs. $2 \%$, respectively), hand-foot syndrome ( $24 \%$ vs. $1 \%$, respectively), alopecia ( $6 \%$ vs. $7 \%$ ), and fatigue/asthenia (8\% vs. $11 \%$, respectively) ${ }^{[17]}$.

Management of capecitabine side effects has recently been reviewed ${ }^{[18]}$. Nab-paclitaxel, a nanoparticle albumin-bound formulation of paclitaxel, was developed as an approach to mitigate some of the disadvantages of the conventional taxane formulations by circumventing the need for the Cremophor EL (CrEL) solvent system ${ }^{[19]}$. In the metastatic breast cancer setting, nab-paclitaxel is indicated as a monotherapy for patients who failed combination chemotherapy for metastatic disease (prior therapy should have included anthracycline unless clinically contraindicated) ${ }^{[20]}$. In a phase 3 trial in patients $(n=454)$ with metastatic breast cancer, nab-paclitaxel (mostly as first- or second-line therapy in the metastatic breast cancer setting) was shown to be more effective and to have a favorable safety profile compared with conventional paclitaxel ${ }^{[21]}$. In the late-line setting, a phase 2 study of nab-paclitaxel in metastatic breast cancer patients heavily pretreated with taxanes evaluated 2 nab-paclitaxel doses $\left(100 \mathrm{mg} / \mathrm{m}^{2}, \mathrm{n}=106\right.$ and $125 \mathrm{mg} / \mathrm{m}^{2}, \mathrm{n}=75$; treatment was given on Days 1, 8, and 15 of a 28-day cycle) and found response rates of $14 \%$ and $16 \%$, respectively; median progression-free survival (PFS) times of 3 months and 3.5 months, respectively; and median OS of 9.2 and 9.1 months, respectively ${ }^{[22]}$. The most common grade $3 / 4$ AEs in this trial included leukopenia (19\% and $36 \%$ for the $100 \mathrm{mg} / \mathrm{m}^{2}$ and $125 \mathrm{mg} / \mathrm{m}^{2}$ doses, respectively) and neutropenia (18\% and 34\%, respectively). No grade 4 non-hematologic AEs were reported for more than 
1 patient. Grade 3 non-hematologic AEs included fatigue (5\% and 12\%, respectively), sensory neuropathy (8\% and 19\%, respectively), nausea ( $4 \%$ and $3 \%$, respectively), diarrhea ( $<1 \%$ and $5 \%$, respectively), and vomiting (3\% and $1 \%$, respectively) ${ }^{[22]}$.

Ixabepilone, a semi-synthetic epothilone analog, is indicated as a monotherapy for patients with locally advanced or metastatic breast cancer who have failed an anthracycline, a taxane, and capecitabine and in combination with capecitabine for the treatment of metastatic or locally advanced breast cancer after a failure of an anthracycline and a taxane ${ }^{[23,24]}$. In a phase 2 single-arm study of ixabepilone monotherapy in patients with advanced breast cancer resistant to an anthracycline, a taxane, and capecitabine, independent radiology facility (IRF)-assessed objective response rate (ORR) in all treated patients $(\mathrm{n}=126)$ was $11.1 \%, 50 \%$ of patients achieved SD, $14.3 \%$ had SD for $\geqslant 6$ months, median PFS was 3.1 months, and median OS was 8.6 months ${ }^{[25]}$. The most common grade 3/4 AEs were neutropenia (54\%), leukopenia (49\%), peripheral sensory neuropathy (14\%), fatigue/asthenia (14\%), anemia (8\%), thrombocytopenia (8\%), myalgia/arthralgia (8\%), and stomatitis/mucositis (7\%) ${ }^{[25]}$. In 2 phase 3 trials comparing ixabepilone plus capecitabine with capecitabine monotherapy in patients with locally advanced/metastatic breast cancer who were pretreated with taxanes and anthracyclines (one study included 752 patients and the other 1,221 patients), the ixabepilone/capecitabine combination significantly improved ORR (35\% vs. $14 \%$ and $43 \%$ vs. $29 \%, P<0.0001$ for both) and median PFS (5.8 vs. 4.2 months; $P=0.0003$ and 6.2 vs. 4.2 months; $P=0.0005$, respectively) ${ }^{[26,27]}$. In both studies, the combination was not associated with a statistically significant improvement in OS (12.9 vs. 11.1 months; $P=0.19$ and 16.4 vs. 15.6 months; $P=0.1162{ }^{[23,27]}$. Notably, a recent pooled analysis performed by Roche and colleagues (2011) evaluated efficacy in these phase 3 trials by Karnofsky's performance status (KPS; high performance status [KPS 90-100] vs. low performance status [KPS 70-80]) ${ }^{[28]}$. The analysis demonstrated that the ixabepilone/capecitabine combination conferred PFS benefits on patients with either high $(\mathrm{n}=1,349)$ or reduced $(\mathrm{n}=606)$ performance status (median PFS: 6.0 vs. 4.4 months, $P=0.0009$, for the ixabepilone/capecitabine combination vs. the capecitabine monotherapy group, in the high-performance status group, and 4.6 vs. 3.1 months; $P=0.0021$, for these 2 groups, respectively, in the reduced-performance group); however, a significant OS benefit was observed only in the reduced-performance group (median OS: 12.3 vs. 9.5 months; $P=0.0015$ ) and not in the high-performance group (16.7 vs. 16.2 months; $P=0.811$ ), suggesting that for certain patient subpopulations, ixabepilone/capecitabine combination may prolong survival ${ }^{[28]}$.

In both phase 3 studies, myelosuppression was observed in the combination arm. Grade 3/4 neutropenia was more common in the combination arm (68\% vs. $11 \%$ and $73 \%$ vs. $9 \%$ ), as well as grade $3 / 4$ leukopenia (57\% vs. $6 \%$ and $63 \%$ vs. $7 \%$ ), anemia ( $10 \%$ vs. $4 \%$ and $5 \%$ vs. $4 \%$ ), and thrombocytopenia ( $8 \%$ vs. $4 \%$ and $6 \%$ vs. $3 \%){ }^{[26,27]}$ Similarly, grade $3 / 4$ peripheral neuropathy was more common in the combination arm (23\% vs. $0 \%$ and $25 \%$ vs. $1 \%)^{[26]}(2129\}$. Other frequently reported non-hematologic grade 3/4 AEs included hand-foot syndrome (18\% vs. $17 \%$ and $21 \%$ vs. $20 \%$ ), fatigue ( $9 \%$ vs. $3 \%$ and $12 \%$ vs. $3 \%$ ), myalgia ( $8 \%$ vs. $0.3 \%$ and $4 \%$ vs. $0 \%$ ), asthenia ( $7 \%$ vs. $0.8 \%$ and $6 \%$ vs. $1 \%$ ), and diarrhea (6\% vs. $\% 9 \%$ and $7 \%$ vs. $9 \%)^{[26,27]}$

Several classes of endocrine therapy are options for patients with heavily pretreated ER-positive metastatic breast cancer, although resistance is likely to develop ${ }^{[6]}$. Up to 3 different endocrine therapies may be attempted if progression or symptomatic visceral disease develops or toxicity is unacceptable ${ }^{[7]}$.

Eribulin, a non-taxane microtubule dynamics inhibitor with a mechanism of action distinct from that of other tubulin-targeting agents ${ }^{[3]}$, has proven well tolerated and efficacious in this hard-to-treat breast cancer population because its distinct mechanism of action may allow it to be active in patients whose disease is refractory to tubulin-targeting agents. Several phase 1 studies of eribulin in patients with advanced solid tumors have demonstrated that eribulin has a manageable safety profile, with neutropenia and febrile neutropenia being the only dose-limiting toxicities observed ${ }^{\text {[29] }}$. $(13079\}^{[30]}$ 
Two single-arm phase 2 studies evaluated eribulin monotherapy. One study was in patients with metastatic breast cancer previously treated with an anthracycline and a taxane $(n=103)^{[31]}$; the other was in patients with locally advanced or metastatic breast cancer previously treated with an anthracycline, a taxane, and capecitabine $(n=291)^{[32]}$. The ORR values were $11.5 \%$ and $9.3 \%$ (all partial responses [PRs]) for these 2 studies, respectively. The SD rates were $41.7 \%$ and $46.5 \%$, respectively, and the clinical benefit rates (defined as complete response [CR] plus PR plus SD for $\geqslant 6$ months) were similar (17.1\% and 17.2\%). The median duration of response (DOR) was 5.6 and 4.1 months (respectively); median PFS was 2.6 months in both studies; and the median OS was 9.0 and 10.4 months (respectively). In both studies, the most common AEs were neutropenia, leukocytopenia, and asthenia/fatigue, with peripheral neuropathy occurring in 5\%-7\% of patients ${ }^{[31,32]}$.

The phase 3 clinical trial for eribulin in metastatic breast cancer (The EMBRACE Trial) was a multicenter, randomized, open-label study; its primary objective was to compare OS for eribulin versus treatment of physician's choice (TPC) in patients $(n=762)$ with locally recurrent or metastatic breast cancer who had received 2 to 5 previous chemotherapy regimens including an anthracycline and a taxane, unless contraindicated (at least 2 regimens for advanced disease) ${ }^{[33]}$ TPC was chosen for the control arm because it reflects "real-life" treatment choices at the time of study enrollment (2006-2008). The majority of patients in the TPC arm (96.4\%) received chemotherapy, most commonly vinorelbine, gemcitabine, or capecitabine. The eribulin arm demonstrated significantly extended OS relative to the TPC arm (median OS, 13.1 vs. 10.7 months; hazard ratio [HR], 0.81; 95\% confidence interval [CI], 0.66-0.99; $P=0.04$, intent-to-treat [ITT] population), making eribulin the first single agent (cytotoxic or biologic) to show survival benefit in this setting (by 2.4 months, representing an increase of $>20 \%$ ). Overall, the findings for the primary endpoint were consistent with those of secondary surrogate endpoints such as ORR and PFS. ORR was $12.2 \%$ with eribulin and $4.7 \%$ with TPC $(P=0.002)$. Of the patients who responded, 3 CRs were observed in the eribulin arm versus none in the TPC arm. Although the difference in median PFS was not statistically significant between the eribulin and TPC arms according to the independent review assessment (3.7 vs. 2.2 months, respectively; HR, 0.87; 95\% CI, 0.71-1.05; $P=0.14$, ITT population), the difference was significant per the investigator assessment (3.6 vs. 2.2 months, respectively; HR, 0.76; 95\% CI, 0.64-0.90; $P=0.002$, ITT population) ${ }^{[33]}$.

The safety profile of eribulin in the EMBRACE study was manageable and consistent with that of earlier studies. The most common grade 3/4 AEs were neutropenia (45.2\% vs. $21.1 \%$ in the eribulin and TPC arms, respectively), leukopenia (13.9\% vs. $5.7 \%$, respectively), and asthenia/fatigue ( $8.8 \%$ vs. $10.1 \%$, respectively). Grade $3 / 4$ peripheral neuropathy occurred in $8.2 \%$ and $2.0 \%$ of patients (eribulin vs TPC, respectively). Although peripheral neuropathy was the most common AE leading to discontinuation of treatment in the eribulin arm (4.8\%), for the patients who did continue eribulin treatment, the peripheral neuropathy typically improved (to grade 2 or better) in later cycles, following dose delays or reductions.30 Other reported AEs included anemia ( $2.2 \%$ vs. $3.6 \%$ in the eribulin and TPC arms, respectively), nausea ( $1.2 \%$ vs. $2.4 \%$, respectively), arthralgia/myalgia ( $0.4 \%$ vs. $1.2 \%$, respectively), and hand-foot syndrome ( $0.4 \%$ vs. $3.6 \%$, respectively) ${ }^{[33]}$.

An analysis of the cost-effectiveness of eribulin relative to the other most commonly used agents in the TPC arm of the EMBRACE study was reported recently ${ }^{[34]}$. Quality-adjusted life-years (QALY) and incremental cost-effectiveness ratios (ICER) were calculated using median OS data of the EMBRACE study. The main drivers of this model were drug acquisition costs, OS, and health utility values. These assessments indicated that eribulin was cost-effective relative to liposomal doxorubicin, nab-paclitaxel, capecitabine, and ixabepilone. Eribulin was marginally cost-effective relative to less expensive drugs, such as vinorelbine and gemcitabine ${ }^{[34]}$.

Eribulin may have advantages compared with other treatment options for heavily pretreated metastatic breast cancer. In particular, eribulin dosing regimens require no premedication to prevent AEs and a short 2- to 5-minute infusion time. These characteristics reduce the time and stress associated with chemotherapy and add potentially to quality of life for these patients. In addition, lower doses than the $1.4 \mathrm{mg} / \mathrm{m}^{2}$ on Days 1 and 8 of a 21-day cycle regimen indicated in 
prescribing information ${ }^{[4]}$ may reduce side effects while remaining efficacious. The patient described here and others in our practice received $1.1 \mathrm{mg} / \mathrm{m}^{2}$, and we also routinely increase the time between the first and second doses to 14 days instead of 7 days, thus giving sufficient time for the patient to recover from potential side effects such as neutropenia. These practical considerations for use in the clinic reduce chemotherapy costs as well as improve patients' quality of life.

\section{Conclusion}

Effective treatment of heavily pretreated patients with metastatic breast cancer remains a challenge. However, newer therapeutic options, particularly the introduction of eribulin to the treatment armamentarium, raises the hope that prolongation of survival, along with maintained/improved quality of life, may be an achievable goal for patients with metastatic breast cancer who had been heavily pretreated, as in the current case study.

\section{Acknowledgments}

The authors wish to acknowledge Philip Sjostedt from The Medicine Group for editorial support.

\section{Conflict of I nterests}

Fariborz Gorouhi has no competing interests to disclose. Stefan Glück has received funding for research and advisory board participation from Eisai, Inc.

\section{References}

[1] Towle MJ, Salvato KA, Budrow J et al. In vitro and in vivo anticancer activities of synthetic macrocyclic ketone analogues of halichondrin B. Cancer Res. 2001; 61(3): 1013-1021. PMid:11221827

[2] Smith JA, Wilson L, Azarenko O et al. Eribulin binds at microtubule ends to a single site on tubulin to suppress dynamic instability. Biochemistry. 2010; 49(6): 1331-1337. PMid:20030375 http://dx.doi.org/10.1021/bi901810u

[3] Jordan MA, Kamath K, Manna T et al. The primary antimitotic mechanism of action of the synthetic halichondrin E7389 is suppression of microtubule growth. Mol Cancer Ther. 2005; 4(7): 1086-1095. PMid:16020666 http://dx.doi.org/10.1158/1535-7163.MCT-04-0345

[4] Halaven [package insert]. Woodcliff Lake, NJ: Eisai Inc.; 2010.

[5] Gluck S, Ross JS, Royce M et al. TP53 genomics predict higher clinical and pathologic tumor response in operable early-stage breast cancer treated with docetaxel-capecitabine +/- trastuzumab. Breast Cancer Res Treat. 2012; 132(3): 781-791. PMid:21373875 http://dx.doi.org/10.1007/s10549-011-1412-7

[6] Roche H, Vahdat LT. Treatment of metastatic breast cancer: second line and beyond. Ann Oncol. 2011; 22(5): $1000-1010$. PMid:20966181 http://dx.doi.org/10.1093/annonc/mdq429

[7] NCCN Clinical Practice Guidelines in Oncology: Breast Cancer. Version 2.2011. 2012.

[8] Jones SE. Metastatic breast cancer: the treatment challenge. Clin Breast Cancer. 2008; 8(3): 224-233. PMid:18650152 http://dx.doi.org/10.3816/CBC.2008.n.025

[9] Gemzar [package insert]. Indianapolis, IN: Eli Lilly; 2012.

[10] Malmstrom A, Hansen J, Malmberg L et al. Gemcitabine and capecitabine in combination for advanced anthracycline and taxane pre-treated breast cancer patients: A phase II study. Acta Oncol. 2010; 49(1): 35-41. PMid:19839920 http://dx.doi.org/10.3109/02841860903268031

[11] Chan D, Yeo WL, Tiemsim CM et al. Phase II study of gemcitabine and carboplatin in metastatic breast cancers with prior exposure to anthracyclines and taxanes. Invest New Drugs. 2010; 28(6): 859-865. PMid:19705063 http://dx.doi.org/10.1007/s10637-009-9305-x

[12] Montero AJ, Talebi TN, Zhu Y et al. Successful use of biweekly gemcitabine plus nab-paclitaxel in two male patients with stage iv breast cancer: case reports and review of the literature. Am J Ther. 2011; 18(1): e12-e18. PMid:20027107 http://dx.doi.org/10.1097/MJT.0b013e3181c219e2

[13] Vinorelbine [package insert]. Research Triangle Park, NC: GlaxoSmithKline. 2002. 
[14] Estevez LG, Batista N, Sanchez-Rovira P et al. A Phase II study of capecitabine and vinorelbine in patients with metastatic breast cancer pretreated with anthracyclines and taxanes. Clin Breast Cancer. 2008; 8(2): 149-154. PMid:18621611 http://dx.doi.org/10.3816/CBC.2008.n.015

[15] Seo HY, Lee HJ, Woo OH et al. Phase II study of vinorelbine monotherapy in anthracycline and taxane pre-treated metastatic breast cancer. Invest New Drugs. 2011; 29(2): 360-365. PMid:19943080 http://dx.doi.org/10.1007/s10637-009-9357-y

[16] Xeloda [package insert]. South San Francisco, CA: Roche. 2012.

[17] O'Shaughnessy J, Miles D, Vukelja S et al. Superior survival with capecitabine plus docetaxel combination therapy in anthracycline-pretreated patients with advanced breast cancer: phase III trial results. J Clin Oncol. 2002; 20(12): $2812-2823$. PMid:12065558 http://dx.doi.org/10.1200/JCO.2002.09.002

[18] Bayraktar S, Gluck S. Management of capecitabline-related gastrointestinal toxicities in women with breast cancer. Commun Oncol. 2011; 8(2): 81-88. http://dx.doi.org/10.1016/S1548-5315(12)70041-4

[19] Di CF, Gasperoni S, Rotella V, Di CF. Targeted delivery of albumin bound paclitaxel in the treatment of advanced breast cancer. Onco Targets Ther. 2009; 2: 179-188.

[20] Abraxane [package insert]. Los Angeles, CA: Abraxis Oncology. 2009.

[21] Gradishar WJ, Tjulandin S, Davidson N et al. Phase III trial of nanoparticle albumin-bound paclitaxel compared with polyethylated castor oil-based paclitaxel in women with breast cancer. J Clin Oncol. 2005; 23(31): 7794-7803. PMid:16172456 http://dx.doi.org/10.1200/JCO.2005.04.937

[22] Blum JL, Savin MA, Edelman G et al. Phase II study of weekly albumin-bound paclitaxel for patients with metastatic breast cancer heavily pretreated with taxanes. Clin Breast Cancer. 2007; 7(11): 850-856. PMid:18269774 http://dx.doi.org/10.3816/CBC.2007.n.049

[23] Ixempra [package insert]. Bistol-Myers Squibb Company: Priceton, NJ. 2010.

[24] Montero AJ, Adams B, Diaz-Montero CM, Gluck S. Nab-paclitaxel in the treatment of metastatic breast cancer: a comprehensive review. Expert Rev Clin Pharmacol. 2011; 4(3): 329-334. PMid:22114779 http://dx.doi.org/10.1586/ecp.11.7

[25] Perez EA, Lerzo G, Pivot X et al. Efficacy and safety of ixabepilone (BMS-247550) in a phase II study of patients with advanced breast cancer resistant to an anthracycline, a taxane, and capecitabine. J Clin Oncol. 2007; 25(23): 3407-3414. PMid:17606974 http://dx.doi.org/10.1200/JCO.2006.09.3849

[26] Thomas ES, Gomez HL, Li RK et al. Ixabepilone plus capecitabine for metastatic breast cancer progressing after anthracycline and taxane treatment. J Clin Oncol. 2007; 25(33): 5210-5217. PMid:17968020 http://dx.doi.org/10.1200/JCO.2007.12.6557

[27] Sparano JA, Vrdoljak E, Rixe O et al. Randomized phase III trial of ixabepilone plus capecitabine versus capecitabine in patients with metastatic breast cancer previously treated with an anthracycline and a taxane. J Clin Oncol. 2010; 28(20): 3256-3263. PMid:20530276 http://dx.doi.org/10.1200/JCO.2009.24.4244

[28] Roche H, Conte P, Perez EA et al. Ixabepilone plus capecitabine in metastatic breast cancer patients with reduced performance status previously treated with anthracyclines and taxanes: a pooled analysis by performance status of efficacy and safety data from 2 phase III studies. Breast Cancer Res Treat. 2011; 125(3): 755-765. PMid:21128114 http://dx.doi.org/10.1007/s10549-010-1251-y

[29] Goel S, Mita AC, Mita M et al. A phase I study of eribulin mesylate (E7389), a mechanistically novel inhibitor of microtubule dynamics, in patients with advanced solid malignancies. Clin Cancer Res. 2009; 15(12): 4207-4212. PMid:19509177 http://dx.doi.org/10.1158/1078-0432.CCR-08-2429

[30] Synold TW, Morgan RJ, Newman EM. A phase I pharmacokinetic and target validation study of the novel anti-tubulin agent E\&389: a California Cancer Consortium trial.[Abstract]. J Clin Oncol. 2005; 23(16s).

[31] Vahdat LT, Pruitt B, Fabian CJ et al. Phase II study of eribulin mesylate, a halichondrin B analog, in patients with metastatic breast cancer previously treated with an anthracycline and a taxane. J Clin Oncol. 2009; 27(18): 2954-2961. PMid:19349550 http://dx.doi.org/10.1200/JCO.2008.17.7618

[32] Cortes J, Vahdat L, Blum JL et al. Phase II study of the halichondrin B analog eribulin mesylate in patients with locally advanced or metastatic breast cancer previously treated with an anthracycline, a taxane, and capecitabine. J Clin Oncol. 2010; 28(25): 3922-3928. PMid:20679609 http://dx.doi.org/10.1200/JCO.2009.25.8467

[33] Cortes J, O'Shaughnessy J, Loesch D et al. Eribulin monotherapy versus treatment of physician's choice in patients with metastatic breast cancer (EMBRACE): a phase 3 open-label randomised study. Lancet. 2011; 377(9769): 914-923. http://dx.doi.org/10.1016/S0140-6736(11)60070-6

[34] Montero AJ, Avancha KK, Gluck S, de Lima Lopez G. Cost-effectiveness analysis of eribulin (E) versus treatment of physician's choice (TPC) in patients (pts) with pretreated metastatic breast cancer (MBC). Breast Cancer Res Treat. http://dx.doi.org/ 10.1007/s10549-012-2326-8 